\title{
Bradyrhizobium sp. Per 3.61: un aporte promisorio para su uso como inoculante en el cultivo de soja
}

Bruno, Carla $\mathrm{V}^{(1)}$, Thuar, Alicia $\mathrm{M}^{(1)}$, Castro, Stella $\mathrm{M}^{(2)}$

${ }^{(1)}$ Departamento de Biología Agrícola, Facultad de Agronomía y Veterinaria. ${ }^{(2)}$ Departamento de Ciencias Naturales, Facultad de Ciencias Exactas, Físico-Químicas y Naturales, Universidad Nacional de Río Cuarto. Río Cuarto. Córdoba. Argentina.

E-mail: cbruno@ayv.unrc.edu.ar

Introducción: En Argentina, la soja representa la mayor superficie cultivada, siendo una de las principales fuentes de ingreso de divisas en los últimos años. Dado que esta leguminosa tiene una gran demanda de nitrógeno $(\mathrm{N})$ y casi su totalidad de grano se exporta, por lo que, si la fijación biológica del nitrógeno (FBN) no es eficiente, junto con ese grano estaremos exportando una parte importante de la fertilidad nitrogenada de nuestros suelos. El objetivo del presente trabajo fue evaluar el impacto de cepas de Bradyrhizobium sp., seleccionadas por poseer una elevada actividad de la enzima nitrato reductasa (NR), sobre el rendimiento del cultivo de soja en presencia de alto contenido de nitrato $\mathrm{NO}_{3}{ }^{-}$en el suelo. Materiales y métodos: El estudio se realizó en el campo experimental (CAMDOCEX) de la Facultad de

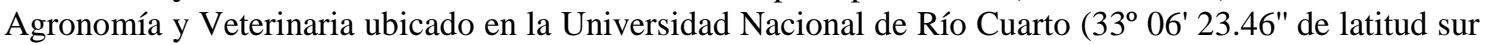
y $64^{\circ} 17^{\prime} 54^{\prime \prime}$ de longitud oeste) en un suelo clasificado como Hapludol típico, con un contenido de 96,60 ppm N-NO ${ }_{3}^{-}(0-20 \mathrm{~cm})$ y $84,20 \mathrm{ppm} \mathrm{N}-\mathrm{NO}_{3}{ }^{-}(20-40 \mathrm{~cm})$. El diseño experimental que se utilizó fue de bloques al azar con tres repeticiones, con los siguientes tratamientos: Control (sin inocular ni fertilizar), Fertilizado (sin inocular y con la adición de $180 \mathrm{~kg} \mathrm{~N}^{-1}$ de urea a la siembra), Inoculado con las cepas de Bradyrhizobium diazoefficiens USDA110 y Bradyrhizobium sp. Per 3.61. Las plantas se cosecharon en la etapa fenológica R2 (plena floración) y R6 (máximo tamaño de semilla) evaluando el Contenido de Nitrógeno: método de Kjeldahl modificado (Nelson y Sommers, 1973); y en la etapa fenológica R8 (plena madurez) se analizó: Balance relativo de nitrógeno (Cholaky y col., 1986). Estimación de Rendimiento (Kantolic y col., 2003). Análisis Estadístico: ANOVA y prueba de Duncan para las comparaciones múltiples de las medias con un nivel de significancia de 5\%.

Resultados: Los resultados obtenidos demostraron que el aporte de FBN de la cepa USDA110 fue del $56 \%\left(215,71 \mathrm{~kg} \mathrm{ha}^{-1}\right)$ y del $64 \%\left(269,14 \mathrm{~kg} \mathrm{ha}^{-1}\right)$ de la cepa Per 3.61, lo cual representa un aumento del $20 \%$ y $39 \%$ del $\mathrm{N}$ acumulado por el cultivo con respecto al tratamiento control. Los datos del rendimiento del cultivo de soja mostraron, que hay diferencias estadísticamente significativas entre los tratamientos inoculados con las diferentes cepas de Bradyrhizobium spp. y fertilizado en relación con el control. El mayor incremento correspondió a las plantas inoculadas con la cepa Per 3.61 mostrando un aumento del $69 \%$ en relación al control y al fertilizado. La respuesta diferencial manifestada en el rendimiento del cultivo soja usando las cepas desnitrificantes de Bradyrhizobium diazoefficiens USDA110 y Bradyrhizobium sp. Per 3.61, ha puesto en evidencia la capacidad de las cepas de mantener la fijación biológica de nitrógeno en presencia de un alto contenido de nitrato.

Conclusiones: En conclusión, es de destacar que la cepa desnitrificante Bradyrhizobium sp. Per 3.61 (caracterizada por llevar a cabo el proceso completo de desnitrificación) representa un aporte promisorio para su uso como inoculante para soja con el fin de mejorar el rendimiento del cultivo. 